\title{
Star Cluster Formation in Extreme Starburst Environments
}

\author{
Richard de Grijs \\ Department of Physics \& Astronomy, The University of Sheffield, Hicks \\ Building, Hounsfield Road, Sheffield S3 7RH, UK
}

\begin{abstract}
The currently available empirical evidence on the star formation processes in the extreme, high-pressure environments induced by galaxy encounters, mostly based on high-resolution Hubble Space Telescope imaging observations, strongly suggests that star cluster formation is an important and perhaps even the dominant mode of star formation in the starburst events associated with galaxy interactions.
\end{abstract}

\section{Gravitational Interaction Induced Star (Cluster) Formation}

Close encounters between gas-rich galaxies often have devastating effects on the state of their interstellar medium (ISM), in particular of the ISM of either the smaller or of the most gas-rich of the interacting galaxies (e.g., de Grijs et al. $2001,2003 \mathrm{a}$ ). The ram pressure caused by the time-varying gravitational potential induced by the interaction causes free-floating giant molecular clouds in the affected galaxy's ISM to collapse and enter a phase of violent star formation. This process is particularly well illustrated by the ongoing interaction between NGC 6745 and its small northern companion galaxy, "NGC 6745c" (cf. de Grijs et al. 2003b), where dynamical and kinematic evidence from $\mathrm{HI}$ observations obtained with the Very Large Array support the scenario that the small companion galaxy has traveled from the south east across the eastern edge of the main galaxy to its current location north of the main galaxy, in its wake leaving a frenzy of very violent and active star, and in particular star cluster, formation.

In de Grijs et al. (2003b) we used the ages, masses and metallicities of the rich young star cluster system in NGC 6745 , based on the analysis of multipassband archival Hubble Space Telescope (HST) observations, to derive its cluster formation history and subsequent evolution. We derive a median age, and thus an estimate of the starburst duration, of $\sim 10 \mathrm{Myr}$. NGC 6745 contains a significant population of high-mass "super star clusters (SSCs)", with masses in the range $6.5 \lesssim \log \left(M_{\mathrm{cl}} / \mathrm{M}_{\odot}\right) \lesssim 8.0$. We caution, however, that these massive SSC candidates may not be gravitationally bound objects, but more diffuse star forming regions or aggregates of multiple unresolved clusters instead. Nevertheless, we measure an effective radius for the most massive object $\left(M_{\mathrm{cl}} \simeq 5.9 \times 10^{8} \mathrm{M}_{\odot}\right)$ of only $R_{\mathrm{eff}} \sim 16 \mathrm{pc}$. However, this object appears very elongated, or may in fact be a double cluster. We should keep in mind, of course, that this high mass estimate is a strong function of the (low) metallicity assumed; if we had assumed solar metallicity for this object, the derived age would have been significantly smaller $(\sim 10-20 \mathrm{Myr}$ vs. $\sim 1 \mathrm{Gyr})$, and the mass could be smaller by a factor of $\gtrsim 10$. Even so, if we could confirm this 
mass estimate spectroscopically, either of the subcomponents would be the most massive cluster known to date, significantly exceeding cluster W3 in NGC 7252, which has a mass of about $(3-18) \times 10^{7} \mathrm{M}_{\odot}$, depending on the age, metallicity and IMF assumed (Schweizer \& Seitzer 1998; Maraston et al. 2001).

The suggestion that such massive objects (either star clusters, or extended star-forming regions) form preferentially in the extreme environments of interacting galaxies, is supported quantitatively by our analysis (de Grijs et al. 2003a) of pixel-by-pixel colour-magnitude and colour-colour diagrams of the Mice and Tadpole interacting galaxies (NGC 4676 and UGC 10214, respectively), based on a subset of the (archival) HST Advanced Camera for Surveys Early Release Observations, which provide a powerful technique to explore and deduce the star and star cluster formation histories of galaxies at moderate distances. In each interacting system we found some 40 bright young star clusters, with a characteristic mass of $\sim 3 \times 10^{6} \mathrm{M}_{\odot}$, which are spatially coincident with blue regions of active star formation in their tidal tails and spiral arms. We showed that star cluster formation is a major mode of star formation in galaxy interactions, with $\gtrsim 35 \%$ of the active star formation in encounters occurring in star clusters. In particular, the tidal tail of the Tadpole system is dominated by blue star-forming regions, which occupy some $60 \%$ of the total area covered by the tail and contribute $\sim 70 \%$ of the total flux in the F475W filter (decreasing to $\sim 40 \%$ in $\mathrm{F} 814 \mathrm{~W}$ ).

Finally, in the nearby (ultra)luminous infrared galaxy NGC 6240, we detect a population of massive young star clusters, with tentative evidence (based on statistical considerations) that the more massive clusters are found closer to the galaxy's double (or perhaps triple) nucleus and the most intense starburst region (Pasquali, de Grijs, \& Gallagher 2003).

Thus, the currently available empirical evidence, mostly based on highresolution $H S T$ imaging observations, strongly suggests that star cluster formation is an important and perhaps even the dominant mode of star formation in the starburst events associated with galaxy interactions.

Acknowledgments. I acknowledge invaluable collaborations with Peter Anders, Nate Bastian, Uta Fritze-v. Alvensleben, Jay Gallagher, Henny Lamers, and Anna Pasquali.

\section{References}

de Grijs, R., O'Connell, R.W., Gallagher, J.S., III 2001, AJ, 121, 768

de Grijs, R., Lee, J.T., Mora Herrera, M.C., Fritze-v. Alvensleben, U., Anders, P. 2003a, New Astron., 8, 155

de Grijs, R., Anders, P., Bastian, N., Lynds, R., Lamers, H.J.G.L.M., O’Neil, E.J., Jr. 2003b, MNRAS, 343, 1285

Maraston, C., Kissler-Patig, M., Brodie, J.P., Barmby, P., Huchra, J.P. 2001, A\&A, 370,176

Pasquali, A., de Grijs, R., Gallagher, J.S., III 2003, MNRAS, 345, 161 (astro-ph/0306446)

Schweizer, F., Seitzer, P. 1998, AJ, 116, 2206 